NASA-CR-200271

\title{
Fountain-Like Flow of Heavy Oxygen Ions From the Earth's Ionosphere in Response to Transverse Heating
}

\author{
Nagendra Singh \\ Department of Electrical and Computer Engineering \\ University of Alabama in Huntsville, Huntsville, AL 35899
}

\begin{abstract}
:
Normally the gravitationally bound heavy $\mathrm{O}^{+}$ions in the Earth's ionosphere are in a diffusive equilibrium. However, when energized to superthermal energies of a few $\mathrm{eV}$ transverse to the geomagnetic field, the combined effects of the-downward gravitational and the upward electric and mirror forces produce interesting flow patterns in the vertical direction like in a pulsating fountain. This flow pattern is studied by means of a particle-in-cell code.
\end{abstract}

It is well known that the topside ionosphere is commony populated with a variety of ion species such as $\mathrm{H}^{+}, \mathrm{O}^{+}$, and $\mathrm{H}_{e}^{+}$. The light ions $\left(\mathrm{H}^{+}\right.$and $\left.\mathrm{H}_{\mathrm{e}}^{+}\right)$readily escape the downward gravitational force even when they have a small characteristic energy of about a few tenths of an $\mathrm{eV}$. On the other hand, for the heavy $\mathrm{O}^{+}$ions the escape requires some sort of energization. For example, an $\mathrm{O}^{+}$ion escapes when its energy $W>W_{e s} \cong m_{O^{+}} g_{o} R_{e}^{2} / r$, where $m_{O^{+}}$is $\mathrm{O}^{+}$ mass, $g_{o}$ is the Earth's gravity at its surface, $R_{e}$ is the Earth's radius, and $r$ is the geocentric distance in the topside ionosphere. Using $R_{e} \cong 6371 \mathrm{~km}, g_{o}=9.8 \mathrm{~ms}^{-2}$ and $r \cong 7350 \mathrm{~km}$, the escape energy $W_{e s} \cong 7.5 \mathrm{eV}$. For $W<W_{e s}$ the $\mathrm{O}^{+}$ions can be transported to large altitudes, but they eventually fall down. If the energization occurs in the degree of freedom parallel to the magnetic field, the falling ions are lost into the ionosphere. On the other hand, when the heating occurs in the degree of freedom transverse to the geomagnetic field, the $\mathrm{O}^{+}$ions are trapped in the flux tube and undergo a bounce motion under the influence of the downward gravitational force and the upward mirror force associated with the downward gradient in the geomagnetic field $\underline{B}$. The upward polarization electric field associated with the density gradient also plays a critical role in the bounce motion. 
We study vertical plasma flow in geomagnetic open flux tubes, which are typical of the polar ionosphere. The topside ionospheric base is assumed to be at $r=r_{b} \cong 7350 \mathrm{~km}$. We simulate the flux tube from $r=r_{b}$ to a geocentric distance $r=r_{\max }=31,350 \mathrm{~km}$. In order to study the plasma flow, we employ a particle code [1]. We inject macroparticles corresponding to $\mathrm{O}^{+}$and $\mathrm{H}^{+}$ions into the flux tube at the boundary at $r=r_{b}$ and subsequently follow their dynamics under the influence of the gravity, polarization electric field and the mirror force associated with the gradient in the geomagnetic field. The injected particles are chosen from Maxweilian velocity distributions with a zero drift for $\mathrm{O}^{+}$and a drift of $2 V_{\text {th }}$ for $\mathrm{H}^{+}$, where $V_{\text {th }}$ is the thermai velocity corresponding to the temperature $T_{0}$ of $\mathrm{H}^{+}$ions at the base. We assume that $\mathrm{O}^{+}$and electron temperatures at $r=r_{b}$ are aiso $T_{o}$. When a steady-state polar wind flow is set up in the flux tube, we initiate the transverse heating event for $\mathrm{O}^{+}$ions at geocentric distances $r \geq 8311 \mathrm{~km}$. The heating at such altitudes is expected to be caused by wave-particle interactions and it is incorporated in a random fashion as described by Brown et al [2]. We present here the flow pattern of $\mathrm{O}^{+}$ions. The $\mathrm{H}^{+}$ions do not show any interesting feature except for their supersonic escape. Since $\mathrm{H}_{e}^{+}$ions have extremely low concentration compared to that of $\mathrm{O}^{+}$and $\mathrm{H}^{+}$, they are not included in the model.

We show the bounce motion of the heated ions and its temporal evolution through a sequence of phase space plots after a brief heating event of 5 minutes at an average rate of about $0.04 k T_{0} / \mathrm{s}$. When $k T_{o} \cong 0.3 \mathrm{eV}$, this leads to a maximum energization of about $15 \mathrm{eV}$. Therefore, some of the ions, having energies $W>W_{e s}$ escape while those with $W<W_{e s}$ are trapped. Figs. $2 \mathrm{a}, 2 \mathrm{~b}$ and $2 \mathrm{c}$ show the initial diffusive state of the $\mathrm{O}^{+}$. Note that the left, middle and right paneis in Fig. 1 are the phase space plots in $r-V_{\|}, r-V_{\perp}$ and $r-W$ planes, where $r$ is the geocentric vertical distance, $V_{\|}$and $V_{\perp}$ are the $\mathrm{O}^{+}$velocity components parallel and perpendicular to the geomagnetic field line, respectively, and $W=\frac{1}{2} m_{o}\left(V_{\|}^{2}+V_{\perp}^{2}\right)$, that is, it is the total kinetic energy. Each dot in the figures represent an $\mathrm{O}^{+}$ion. Figures $2 \mathrm{a}, 2 \mathrm{~b}$ and $2 \mathrm{c}$ show that the buik of $\mathrm{O}^{+}$ions are generally confined below an altitude $r \cong 10^{4} \mathrm{~km}$. 
The state of the $\mathrm{O}^{+}$flow after 6 hours of the brief heating period is shown in Figs 2d, 2e and $2 f$. Note that some ions have reached near the top of the simulated flux tube and have $V_{\|}>0$, implying that they will escape. While those having $V_{\|}<0$ are falling. Those ions are transported to high aititudes by the mirror force $F_{m} x-\frac{1}{2} m_{o} V_{\perp}^{2} B^{-1}(\partial B / \partial r)$. The transverse heating increases $V_{\perp}$ and enables the upward flow. But the gravity pulls them downward if they do not have sufficientily large energy to escape.

The falling ions mirror back and expand upward and they appear in clumps; the clumps are seen in the form of evolving bands in the $r-V_{\|}$plane as seen from Figs $2 \mathrm{~d}, 2 \mathrm{~g}$, and $2 \mathrm{j}$. The bands give the appearance of a water fountain. The bands can be seen as clusters of ions in the $r-V_{\perp}$ plane. $V_{\perp}$ decreases with increasing $\mathrm{r}$ due to the conservation of the magnetic moment $\mu=\frac{1}{2} m V_{\perp}^{2} / B$. In terms of energy, the ion clusters appear in the form of "bananas," which simply manifest that the upgoing ions have slightly more kinetic energy than the downflowing ions. In both $r-V_{\perp}$ and $r-W$ planes, the upflowing $\left(V_{\|}>0\right)$ ions are shown in green while the downflowing are shown in blue.

The ciuster formation occurs by the modulation in the electric field $E$ due to the falling and rising $\mathrm{O}^{+}$ions because $E$ depends on the density gradients. This modulation of $E$ also causes the difference in the kinetic energies of the upgoing and downgoing ions in any given cluster.

Acknowledgment: This work was performed under the grant NAGW-2903.

\section{References}

[1] G. R. Wilson, C. W. Ho, J. L. Horwitz, N. Singh, and T. E. Moore, Geophys. Res. Lett., 17, 263,1990 .

[2] D. G. Brown, G. R. Wilson, J. L. Horwitz, and D. Gallagher, Geophys. Res. Lett., 18, 1841, 1991. 


\section{Figure Captions}

Fig. 1. Phase space plots in $r-V_{\|}$(left panels), $r-V_{\perp}$ (middle panels), and $r-W$ (right paneis) at several times are shown. Note that the velocity components are normalized with respect to the thermal velocity of $\mathrm{H}^{+}$ions which is about $5.4 \mathrm{~km} / \mathrm{s}$ for $k T_{o}=0.3 \mathrm{eV}$ and energy is normalized in terms of $k T_{0}$. In the middle and right paneis, blue represents falling $\mathrm{O}^{+}$ ions, while green represents upflowing ones. 